\title{
Diabetic foot monitoring using dynamic thermography and Al classifiers
}

\author{
by R. Vardasca*, C. Magalhaes*, A. Seixas ${ }^{* *}$, R. Carvalho***, J Mendes* \\ *LABIOMEP, INEGI-LAETA, Faculdade de Engenharia, Universidade do Porto, Rua Dr. Roberto Frias 4200-465 \\ Porto, Portugal \\ ${ }^{*}$ Escola Superior de Saúde, Universidade Fernando Pessoa, Rua Delfim Maia, 334, 4200-253 Porto, Portugal \\ Porto, Portugal \\ ***Serviço de Endocrinologia, Centro Hospitalar e Universitário do Porto, Largo do Prof. Abel Salazar, 4099-001
}

\begin{abstract}
Diabetes Mellitus (DM) is one of the most growing burdens in healthcare, one of its impacting consequences is Diabetic Foot Ulcers (DFU), which will affect at least 1 in each 4 DM patients in their lifetime. If not identified early, DFU can become chronic and in more severe cases lead to amputations affecting seriously the quality of life of patients and increase the healthcare costs. Infrared thermal (IRT) imaging has been used as a research method to early identification of DFU ${ }_{2}$ since an elevation of skin temperature is a sign of inflammation and a reduction a sign of poor vascularization. There are two main types of DFU: neuro-ischemic and ischemic. A database with dynamics IRT plantar foot examination images of 39 active DFU patients was built, the images were analyzed through measuring mean temperature of regions of interest (ROI), which correspond to most frequent documented locations of DFU. Statistics showed that there was no evidence of significant differences between thermal asymmetry values and thermal recovering differences in all ROI, apart from the one located at the medial forefoot. The ROls were assessed in both feet and the value of thermal asymmetry was taken in consideration per each ROI. Using the database with the analysis results, a decision support system was built implementing machine learning algorithms such as: Artificial Neural Networks (ANN), Support Vector Machines (SVM) and k-Nearest Neighbour (k-NN), to classify the data and assess the correct identification of the type of DFU. The best overall result achieved (Table 1) was with k-NN of 5 neighbors with $81.25 \%$ accuracy, $80 \%$ specificity and $100 \%$ sensitivity. These results are promising for DFU early identification and expected to improve with a larger sample.
\end{abstract}

\section{Introduction}

Diabetes Mellitus (DM) is a chronic disease with a growing worldwide incidence, resulting in high burden to individuals and the society [1], being characterized by elevated blood glucose levels in the system. According to the World Health Organization (WHO), in 2014 the global prevalence of DM among adults has risen to $8.5 \%$, affecting near 422 million people, being a direct cause for 1.5 million deaths in 2012 [2] and is expected to become the seventh leading cause of death in 2030 [3].

One of the major consequences of DM is Diabetic Foot Ulcers (DFU), which will affect at least 1 in each 4 DM patients in their lifetime. If not identified early, DFU can become chronic and in more severe cases lead to amputations, due to reduced blood flow and nerve damage [2], affecting seriously the quality of life of patients.

To cope with this growing concern, emerging technologies are required to help DFU early identification, one of the methods proposed is the monitoring of plantar feet skin temperature [4], which can be recorded with infrared thermal (IRT) imaging, either by comparing hot or cold spots with the main value of the feet or by asymmetries with the bilateral limb [5], a threshold of $2.2^{\circ} \mathrm{C}$ was established in previous research using static IRT [6].

Data automated classification algorithms are based in machine learning and statistics are able to category or class each element of a dataset based in features. Supervised algorithms are based in training data, which contains dataset elements with known membership category. These classification algorithms are useful for extracting information.

Examples of these algorithms are:

- $\quad$ Artificial Neural Networks (ANNs) are parallel-distributed information processing structures which model the functionality of small biological neural clusters and thereby mimicking human decision making [7]. Some ANNs are trained with backpropagation algorithm [8]. The ANN algorithms can achieve a fast and low processing cost decision and suitable for hard real-time systems.

- $\quad$ k-Nearest Neighbour ( $k-N N$ ) is a very simple classifier which determines the $k$ nearest neighbors for a specific query instance by calculating the minimum distance to the training samples [9, 10]. A data point is classified based in the majority of votes from these $\mathrm{k}$ neighbors. If $\mathrm{k}$ is 1 , then the data point is assigned to the class of the nearest neighbor. Contributions are based more in closeness of data points.

- $\quad$ Support Vector Machine (SVM) are based in searching for a hyperplane, which subsequently serves as a decision surface. This surface separates the datapoints, which belong to distinct classes, from each other with maximum margin. This classifier is known by high classification accuracy even under adverse conditions, such as nonlinear and high dimensional datasets [11].

A computer assisted tissue classification (granulation, necrotic, and slough) scheme [12] for chronic wound evaluation using medical image processing and statistical machine learning techniques on wound images was built and achieved an overall accuracy of $87.61 \%$, with highest kappa statistic value $(0.793)$, the method used was support vector 
machine (SVM) with a third order polynomial kernel. A system [13] was built with an accuracy of $96.1 \%$ automated detection and classification of DFU using Convolutional Neural Networks in foot images, transforming it in an innovative technique for DFU evaluation and medical treatment.

Liu et al. [14] addressed the image processing and pattern analysis needed for the detection of complications in diabetic foot using IRT images, although their approach might be time and computational consuming, which would make it difficult to implement in daily practice.

A computer assisted diagnosis system [15] was built for diabetic foot detection to prevent traumatic outcomes such as ulcerations and lower extremity amputation. Plantar foot thermograms of 33 healthy persons and 33 individuals with type 2 diabetes were taken. The images were decomposed using discrete wavelet transform (DWT) and higher order spectra (HOS) techniques, which were ranked using t-values and classified using a support vector machine (SVM) classifier, achieving a maximum accuracy of $89.39 \%$, sensitivity of $81.81 \%$ and specificity of $96.97 \%$ using only five features.

The aim of this research is to use IRT images during a dynamic examination by cooling both feet and apply simulated advanced data analysis and Machine Learning (ML) classification algorithms to automatize the procedure of aiding on the early identification of DFU.

\section{Materials and methods}

A database with IRT images from plantar foot images during a dynamic cooling challenge (baseline and during 5 minutes after cooling by conduction with a board of aluminium for 2 minutes, see Fig. 1) of 39 active DFU patients was used. The images were recorded in a controlled environment and followed the internationally accepted guidelines for IRT in medicine $[16,17,18]$.

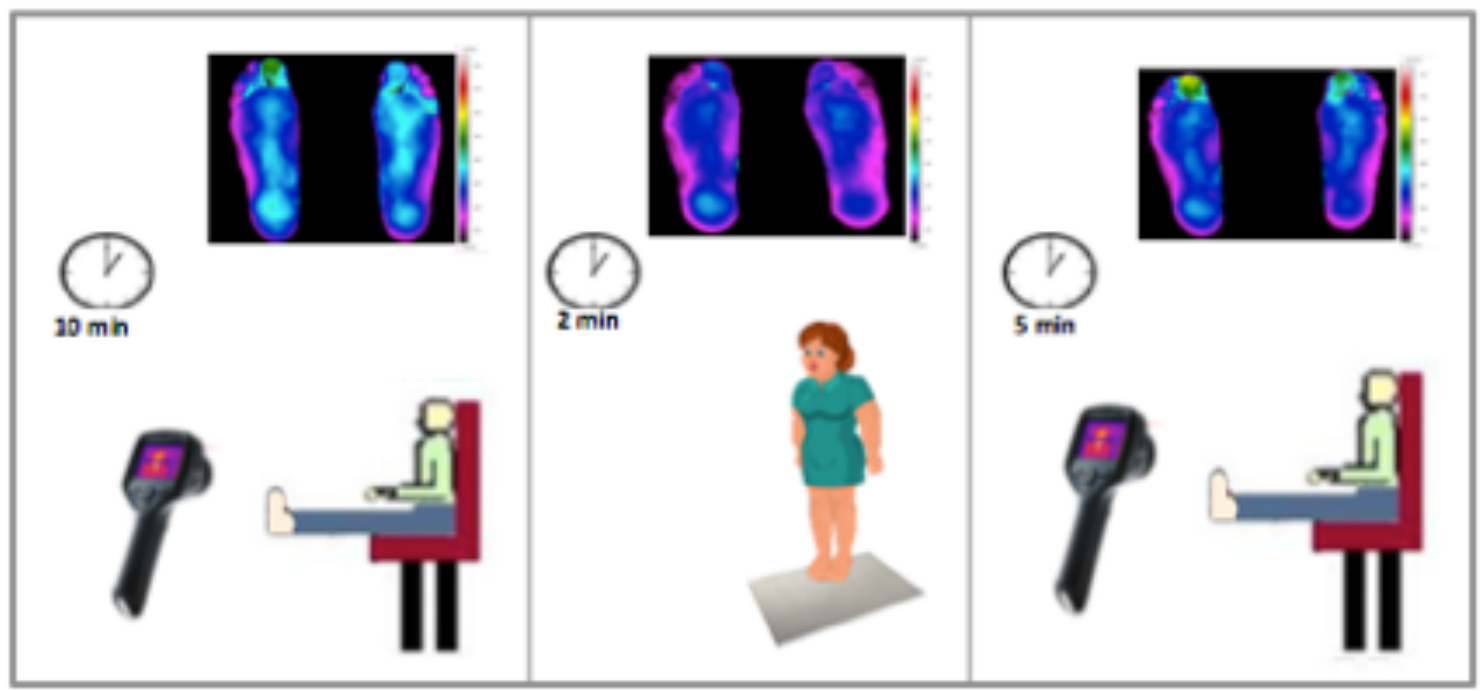

Fig. 2: The procedure of IRT image capture of the plantar feet, acclimatization period of 10 minutes, 2 minutes of provocation and 5 minutes of recovery.

From these 39 patients, only 14 had infected or ischaemic wound, the other 25 had a healing wound. The proposed locations with more prominent locations of DFU formation was used as a reference (Fig. 2) [19]. The locations of the infected or ischaemic wounds were at P1(2), P2(1), P3(1), P6(2), P7(6), P8(2) and P10(1).

The images were taken at Centro Hospitalar e Universitario do Porto with a FLIR® (Wilsoville, OR, USA) E60 thermal camera (FPA sensor array size of $320 \times 240$, NETD of $<50 \mathrm{mK} @ 30^{\circ} \mathrm{C}$, measurement uncertainty of $\pm 2 \%$ of the overall temperature reading). The study was approved by the hospital ethical committee and the participants signed and informed consent. For the image analysis, a specific software was built, which analysed them through measuring mean temperature of regions of interest (ROI) were the DFU had more prominence to appear [19]. 

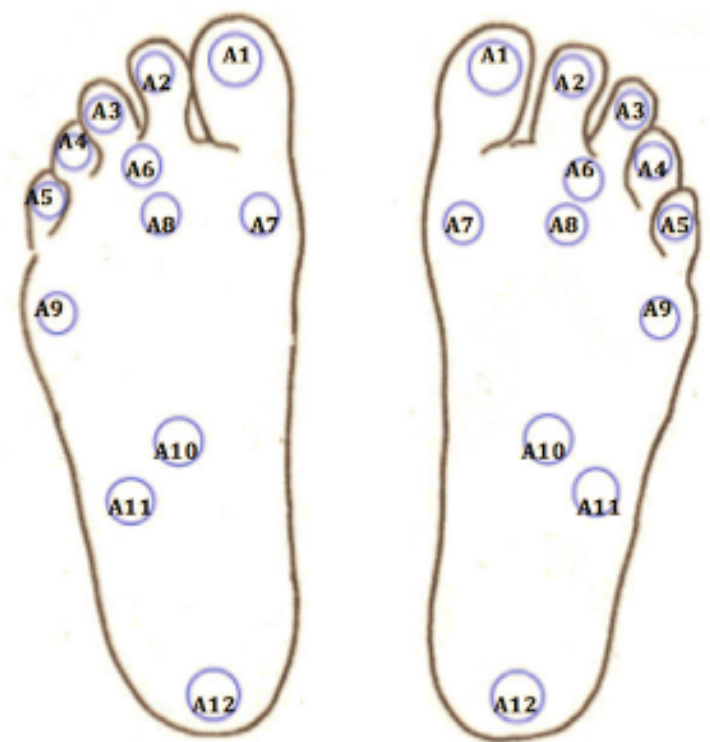

Fig. 2: The plantar feet ROls located at the plantar feet in the areas more prominent to DFU formation.

Non parametric statistical test Mann-Whitney $U$ was used to assess whether there was an influence of having an infected or ischaemic DFU in the temperature measurements, globally and at each location.

The ROI data was extracted from a built software (Fig. 3) that extracted it to an Excel spreadsheet, which was then loaded in WEKA data analysis framework [20] to obtain the classification results (using the Artificial Neural Networks (ANN), Support Vector Machines (SVM) and k-Nearest Neighbour (k-NN) ML classifiers), statistics were also evaluated using the software package IBM SPSS v24.

The obtained classification results were evaluated by performance measures, such as True Positive (TP), True Negative (TN), False Positive (FP), False Negative (FN) Accuracy (A), Sensitivity (SN) and Specificity (SP). The selection of the most appropriate algorithm was based in these measures, which were calculated in the following manner:

- TN - correctly rejected

- $\quad \mathrm{FN}=$ incorrectly rejected

- $\quad T P=$ correctly identified

- $\quad F P=$ incorrectly identified

- $A=(T P+T N) /(T P+F N+T N+F P)$

- $\quad \mathrm{SN}=\mathrm{TP} /(\mathrm{TP}+\mathrm{FN})$

- $\quad \mathrm{SP}=\mathrm{TN} /(\mathrm{TN}+\mathrm{FP})$

- $\quad$ Accuracy $=(T P+T N) /(T P+T N+F P+F N)$

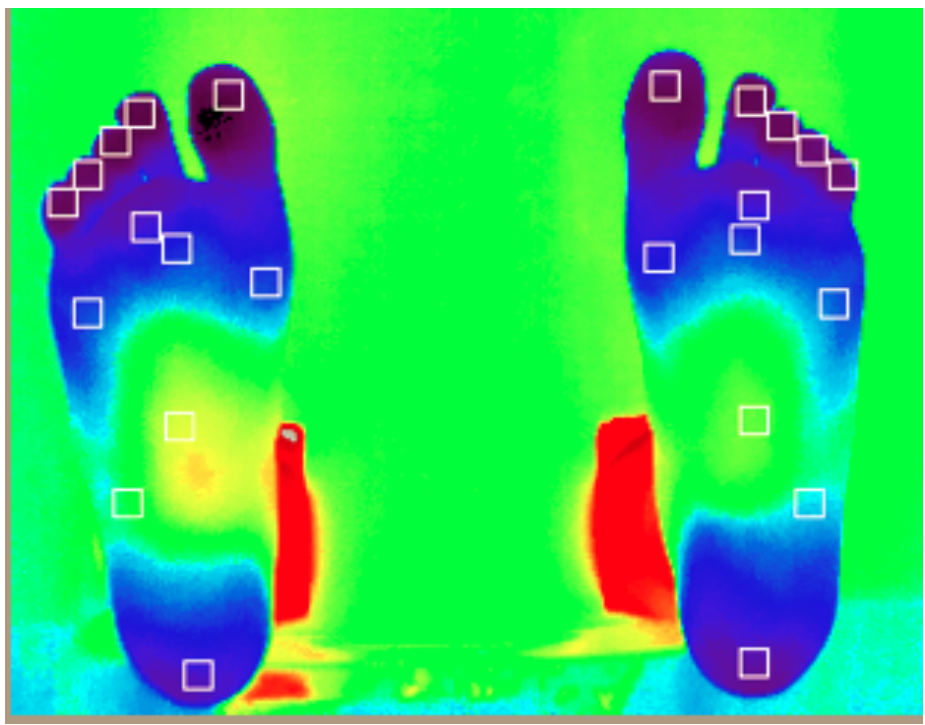

Fig. 3: The plantar feet ROls located as overlay in the developed software. 


\section{Results}

Based in the ROI mean temperatures there was only statistical evidence of discrimination $(p<0.05)$ in the ROI A7 at baseline image and immediately after cooling and at ROI A6 at the asymmetry value of the image, 5 minutes after provocation. When comparing the active infection or ischaemic with the healing ulcers, at mean temperature there is only statistical evidence $(p<0.05)$ at $P 7$ right foot after cooling, although there are also for $P 8, P 10$ and $P 11$ a thermal asymmetry at the moments before cooling and 5 minutes after cooling, and on ROIs P6, P7 and P9 thermal asymmetry at the three moments: before cooling, immediately after cooling and 5 minutes after provocation.

The collected data was analysed with WEKA analysis framework [20], where the ML classifiers were applied and the best accuracy obtained was with the SVM, accuracy with the k-NN ( 5 neighbours) and the best specificity was achieved with both SVM and k-NN (5 neighbours), the overall result was obtained by k-NN (5 neighbours) because of SVM poor sensitivity, as can be seen at table 1 .

Table 1. The ML classification results on the early identification of DFU.

\begin{tabular}{|l|c|c|c|}
\hline Classifier & Accuracy & Sensitivity (SN) & Specificity (SP) \\
\hline ANN & $81.25 \%$ & $75 \%$ & $83 \%$ \\
\hline SVM & $87.5 \%$ & $50 \%$ & $100 \%$ \\
\hline k-NN (5 neighbors) & $81.25 \%$ & $80 \%$ & $100 \%$ \\
\hline
\end{tabular}

\section{Discussion}

The most affected ROI in the studied sample was the A7 - hallux metatarsus, this is shown by statistics, this was the first classification using dynamic IRT data, the achieved results are in line with those obtained from static IRT examinations $[5,15]$ and with other imaging techniques $[12,13]$ but with more diversity of data for further improvements. The whole methodology can be improved and automatized through the methods proposed by Liu et al. [14].

The statistical evidences found at ROls P9 and P11, which did not present active infection or ischaemic might be an early identification of something happen in the healing process.

Despite the promising results, this had been tested in a very small sample, a larger sample and with major variability is required to prove the full contribution of this concept of computer assisted diagnosis system.

\section{Conclusion}

It is possible to identify early DFU by using IRT information and Al classifiers combined with $81.25 \%$ accuracy, $80 \%$ specificity and $100 \%$ sensitivity, and this expected to improve with a larger training sample is required and the usage of more features than only ROls Mean temperatures, ROI recovery thermal gradients or ROls thermal asymmetries. A larger sample is required to prove this methodological concept.

\section{ACKNOWLEGMENTS}

Authors gratefully acknowledge the funding of project LAETA - UID/EMS/50022/2013.

\section{REFERENCES}

[1] Boulton AJ, Vileikyte L, Ragnarson-Tennvall G, Apelqvist J. The global burden of diabetic foot disease. Lancet 2005;366(9498):1719-1724. https://doi.org/10.1016/S0140-6736(05)67698-2

[2] WHO. Global report on diabetes. Geneva. 2016. https://apps.who.int/iris/bitstream/handle/10665/204871/9789241565257_eng.pdf

[3] Mathers CD, Loncar D. Projections of global mortality and burden of disease from 2002 to 2030. PLOS Medicine 2006;3(11):e442. https://doi.org/10.1371/journal.pmed.0030442

[4] Lavery LA, Armstrong DG, Vela SA, Quebedeaux TL, Fleischli JG. Practical criteria for screening patients at high risk for diabetic foot ulceration. Arch. Intern. Med. 1998;158(2):157-162. https://doi.org/10.1001/archinte.158.2.157

[5] Adam M. et al. Automated characterization of diabetic foot using nonlinear features extracted from thermograms. Infrared Physics \& Technology 2018;89:325-337. https://doi.org/10.1016/j.infrared.2018.01.022

[6] Macdonald A. et al. Thermal symmetry of healthy feet: a precursor to a thermal study of diabetic feet prior to skin breakdown. Physiol Meas 2017;38(1):33-44. https://doi.org/10.1088/1361-6579/38/1/33

[7] Patel JL, Goyal RK. Applications of artificial neural networks in medical science. Current clinical pharmacology 2007;2(3):217-226 https://doi.org/10.2174/157488407781668811

[8] Chauvin Y, Rumelhart DE. Backpropagation: theory, architectures, and applications. Psychology Press 2013 
[9] Altman NS An introduction to kernel and nearest-neighbor nonparametric regression. The American Statistician 1992;46(3):175-185

[10] Keller JM, Gray MR, Givens JA. A fuzzy k-nearest neighbor algorithm. IEEE transactions on systems, man, and cybernetics 1985;4:580-585 https://doi.org/10.1109/TSMC.1985.6313426

[11] Wang L. Support vector machines: theory and applications. Springer Science \& Business Media 2005

[12] Mukherjee R, Manohar DD, Das DK, Achar A, Mitra A, Chakraborty C. Automated tissue classification framework for reproducible chronic wound assessment. BioMed research international 2014 http://dx.doi.org/10.1155/2014/851582

[13] Goyal M, Reeves ND, Davison AK, Rajbhandari S, Spragg J, Yap, MH. Dfunet: Convolutional neural networks for diabetic foot ulcer classification. IEEE Transactions on Emerging Topics in Computational Intelligence 2018 http://dx.doi.org/10.1109/TETCI.2018.2866254

[14] Liu C, van Netten JJ, Van Baal JG, Bus SA, van Der Heijden F. Automatic detection of diabetic foot complications with infrared thermography by asymmetric analysis. Journal of biomedical optics 2015;20(2):026003 https://doi.org/10.1117/1.JBO.20.2.026003

[15] Adam M, Ng EY, Oh SL, Heng ML, Hagiwara Y, Tan JH, Tong JWK, Acharya UR. Automated characterization of diabetic foot using nonlinear features extracted from thermograms. Infrared Physics \& Technology 2018;89:325-337 https://doi.org/10.1016/j.infrared.2018.01.022

[16] Ring EFJ, Ammer K. The technique of infrared imaging in medicine. Infrared Imaging. loP Publishing. 2015. https://doi.org/10.1088/978-0-7503-1143-4ch1

[17] Ring EFJ, Ammer K. The technique of infrared imaging in medicine. In Infrared Imaging: A casebook in clinical medicine. loP Publishing 2015 https://doi.org/10.1088/978-0-7503-1143-4ch1

[18] Ammer K. The Glamorgan Protocol for recording and evaluation of thermal images of the human body. Thermology International 2018;18(4):125-129

[19] Vardasca R, Marques A, Carvalho R, Gabriel J. Thermal imaging of the foot in different forms of diabetic disease. Infrared Imaging: A casebook in clinical medicine. IoP Publishing. 2015. https://doi.org/10.1088/978-07503-1143-4ch27

[20] Hall M. et al. The WEKA data mining software: an update. ACM SIGKDD explorations newsletter 2011;11(1):1018. https://doi.org/10.1145/1656274.1656278 